\title{
A BIO-INSPIRED LEARNING AND CLASSIFICATION METHOD FOR SUBCELLULAR LOCALIZATION OF A PLASMA MEMBRANE PROTEIN
}

\author{
Wafa Bel Haj Ali ${ }^{2}$, Paolo Piro ${ }^{1}$, Lydie Crescence ${ }^{3}$, Dario Giampaglia ${ }^{2}$, \\ Oumelkheir Ferhat $^{3}$, Jacques Darcourt ${ }^{3}$, Thierry Pourcher ${ }^{3}$ and Michel Barlaud ${ }^{2}$ \\ ${ }^{1}$ Italian Institute of Technology (IIT), Genoa, Italy \\ ${ }^{2}$ I3S/CNRS Laboratory, University of Nice-Sophia Antipolis, France \\ ${ }^{3}$ Team Tiro CEA/University of Nice-Sophia Antipolis /CAL \\ paolo.piro@iit.it, \{belhajal, giampagl,barlaud\}@i3s.unice.fr, \{crescence,ferhat,darcourt, pourcher\}@unice.fr
}

Keywords: Cell classification, sodium iodide symporter, bio-inspired, $k$-NN, boosting, machine learning.

\begin{abstract}
High-content cellular imaging is an emerging technology for studying many biological phenomena. statistical analyses on large populations (more than thousands) of cells are required. Hence classifying cells by experts is a very time-consuming task and poorly reproducible. In order to overcome such limitations, we propose an automatic supervised classification method. Our new cell classification method consists of two steps: The first one is an indexing process based on specific bio-inspired features using contrast information distributions on cell sub-regions. The second is a supervised learning process to select prototypical samples (that best represent the cells categories) which are used in a leveraged $k$-NN framework to predict the class of unlabeled cells. In this paper we have tested our new learning algorithm on cellular images acquired for the analysis of changes in the subcellular localization of a membrane protein (the sodium iodide symporter). In order to evaluate the automatic classification performances, we tested our algorithm on a significantly large database of cellular images annotated by experts of our group. Results in term of Mean Avarage Precision (MAP) are very promising, providing precision upper than $87 \%$ on average, thus suggesting our method as a valuable decision-support tool in such cellular imaging applications. Such supervised classification method has many other applications in cell imaging in the areas of research in basic biology and medicine but also in clinical histology.
\end{abstract}

\section{INTRODUCTION}

High-content cellular imaging is an emerging technology for studying many biological phenomena. Related cellular image analysis generally requires to classify many cells according to their morphological aspect, staining intensity, subcellular localization and other parameters. Studied biological phenomena can be heterogenous. For example, protein subcellular localisation could depend on the expression of other proteins or the cell states. In this case, statistical analyses on large populations (more than thousands) of cells are required. Furthermore, if time-lapse experiments or drug screenings have to be performed numerous different conditions have to be tested. New powerful fully motorized microscopes are now able to produce thousands of multiparametric images for several experimental conditions. Consequently, large numbers of cell images have to be analysed.

Unfortunately, humans are limited in their ability to classify due to the huge amount of image data and this makes the classification a burdensome task.

To circumvent this, we developed a new classification method for the analysis of the staining morphology of thousands (millions) of cells. First a fast multiparametric image segmentation algorithm extracts cells with their nucleus. Next, our cell classification method consists of two steps: The first one is an indexing process based on specific bio-inspired features using contrast information distributions on cell sub-regions. The second is a supervised learning process to select prototypical samples (that best represent the cells categories) which are used in a leveraged $k$ NN framework to predict the class of unlabeled cells. Such classification method has many applications in cell imaging in the areas of research in basic biology 
and medicine but also in clinical histology.

In the present work, we used our classification method to study the pathways that regulate plasma membrane localization of the sodium iodide symporter (NIS for Natrium Iodide Symporter). NIS is the key protein responsible for the transport and concentration of iodide from the blood into the thyroid gland. NIS-mediated iodide uptake requires its plasma membrane localization that is finely controlled by poorly known mechanisms. For decades, the NIS-mediated iodide accumulation observed in thyrocytes has been a useful tool for the diagnosis (thyroid scintiscan) and treatment (radiotherapy) of various thyroid diseases. Improvements in radioablation therapy might result from promoting targeting of NIS to the plasma membrane in the majority of thyroid cancers or metastases. NIS has also been described as a promissing therapeutic transgene promoting metabolic radiotherapy (i.e., 131I uptake by cancer cells ectopicallyexpressing NIS) in many different studies. An important improvement of this approach should benefit from a better understanding of the post-transcriptional regulation of NIS targeting to the plasma membrane, Previously, we observed that mouse NIS catalyses higher levels of iodide accumulation in transfected cells compared to its human homologue. We showed that this phenomenon was due to the higher density of the murine protein at the plasma membrane. To reach this conclusion, biologists classified several hundreds of cells (Dayem et al., 2008). We have also demonstrated, using a set of monoclonal antibodies, that human NIS is not expressed intracellularly in thyroid and breast cancer (Peyrottes et al., 2009), as was proposed by other groups. Our team is now focussing on the analysis of NIS phosphorylation that most probably plays an important role in the post-transcriptional regulation of the NIS. Using site-directed mutagenesis of previously-identified consensus sites, we have recently shown that direct phosphorylation of NIS alters NIS targeting to the plasma membrane, as well as NIS recycling, causing retention of the protein in intracellular compartments such as the Golgi apparatus, the endoplasmic reticulum or the early endosomes. We used a high-content cellular imaging to study the impact of the mutation of several putative phosphorylation sites on the subcellular distribution of the protein.

\section{CLASSIFICATION METHOD}

Our method for automatic classification of cell images is depicted as a block diagram in Fig. 1. The first step is a pre-processing segmentation of cells from the

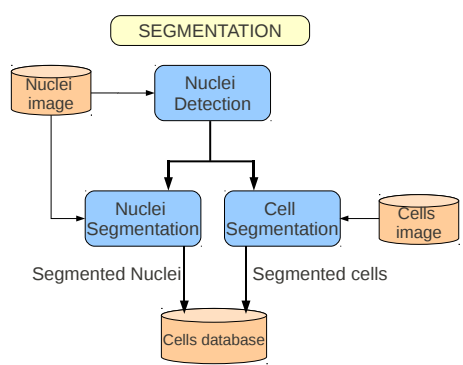

(a)

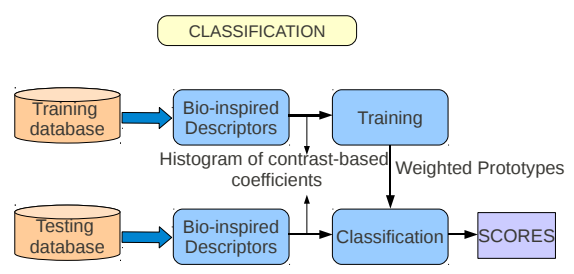

(b)

Figure 1: Block diagram of the proposed method for automatic cell classification: (a) cell segmentation step and (b) descriptor extraction and classification process.

images. The database consist of two distinct parametric fluorescence images. The first one, called nucleus image shows the nucleus and the second called global image, shows the staining of the proteine. Nucleus locations are detected from the nucleus image and used as a prior for cell segmentation of the global image. An example of both images and their segmentation is shown in Fig. 2. Once cells are extracted, we apply our classification method ; First we compute bioinspired region descriptors, extracting contrast-based features for each of the segmented cells. These descriptors are then used in a supervised learning framework where the most relevant prototypical samples are used to predict the class of unlabeled cells.

We split this section in two parts: the first describes our feature extraction approach, whereas the latter is focused on our prototype-based learning algorithm.

\subsection{Region based bio-inspired descriptor}

For better level of performance in differentiating between cells, it can be useful to get inspiration from the way our visual system operates to analyze and represent the visual input. The first transformation under- 


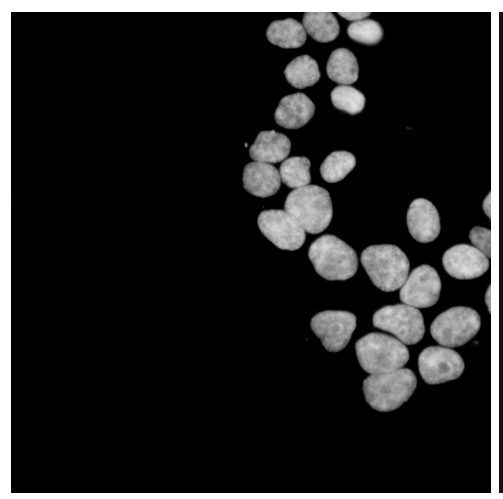

(a)

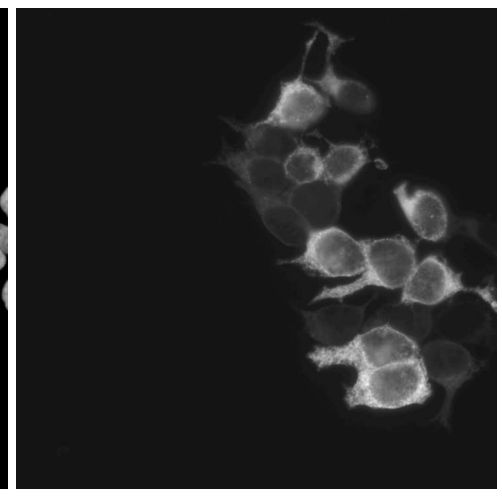

(b)

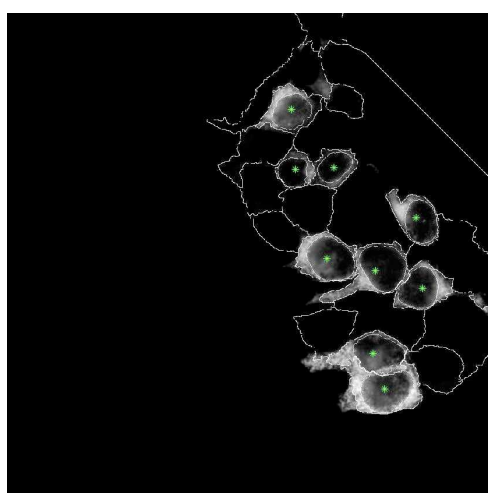

(c)

Figure 2: Image of the nucleus-staining of representative cells (a), NIS-specific immunostaining of the corresponding cells (b) and their segmentation (c).

gone by a visual input is performed by the retina. Inspired by the basic step of a retinal model, we define bio-inspired features for image representation.

The basic idea is to use a descriptor inspired from visual system model and specially from the main characteristics of the retina processing. In fact, the retinal cells are in a first stage sensitive to local differences of illumination. This can be modeled by the luminance contrast as for the BIF descriptors in (Bel haj ali et al., 2011). Such descriptor is well adapted in the case of our cells images since the most discriminative visual feature between categories is the contrast intensity of each region of the cell. Thus, we define cell descriptors based on the local contrast in regions of interest of each cell (nucleus, membrane and cytoplasm). The local contrast is obtained by a filtering with Differences of Gaussians (DoGs) centered at the origin. So that the contrast $\mathcal{C}_{I m}$ for each position $(x, y)$ and a given scale $s$ in the image Im is as follows:

$$
\mathcal{C}_{\text {Im }}(x, y, s)=\sum_{i} \sum_{j}\left(\operatorname{Im}(i+x, j+y) \cdot \operatorname{DoG}_{s}(i, j)\right) .
$$

We used the DoGs described by (Field, 1994) where the larger Gaussian has three times the standard deviation of the smaller one. After computing these contrast coefficients in (1), we apply a non-linear bounded transfer function, named neuron firing rates, used in (Van Rullen and Thorpe, 2001). This function is written as:

$$
R(C)=G \cdot C /(1+\operatorname{Ref} \cdot G \cdot \mathcal{C}),
$$

where $G$ is named the contrast gain and $R e f$ is known as the refractory period, a time interval during which a neuron cell reacts. The values of those two parameters proposed in (Van Rullen and Thorpe, 2001) to best approximate the retinal system are $G=2000 \mathrm{~Hz}$. contrast $^{-1}$ and $\operatorname{Ref}=0.005 \mathrm{~s}$.

To extract our descriptors, we need to define masks on cell images on which we encode firing rate coefficients $R(C)$. According to the visual aspect of cells, we split each cell into two regions of interest as shown in Fig. 3, corresponding to nucleus and external part, by using simple morphological operators. For both of them, firing rate coefficients are quantified into normalized $\mathcal{L} 1$ histograms of 32-bins then concatinated, thus giving our global descriptor with a dimension equal to 64 .
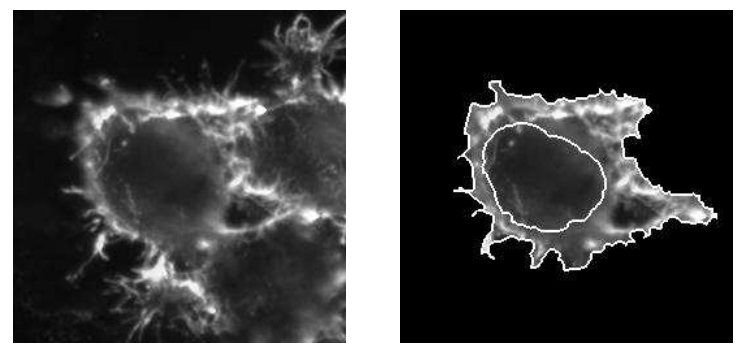

(a)
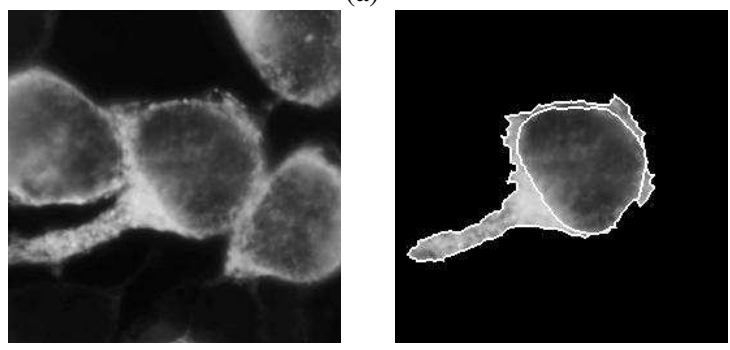

(b)

Figure 3: An $M b$ (a) and an $E R$ (b) extracted cells and their two segmented regions of interest.

Note that state of the art classical methods such as SIFT descriptor encode gradient directions on square blocks (Lowe, 2004) and Gist features encode average energies of filters coefficients on square blocks too (Oliva and Torralba, 2001). 


\subsection{Prototype-based learning}

We consider the multi-class problem of automatic cell classification as multiple binary classification problems in the common one-versus-all learning framework (Schapire and Singer, 1999). Thus, for each class $c$, a query image is given a positive (negative) membership with a certain confidence (classification score). Then the label with the maximum score is assigned to the query.

We suppose given a set $\mathcal{S}$ of $m$ annotated images. Each image is a training example $(\boldsymbol{x}, \boldsymbol{y})$, where $\boldsymbol{x}$ is the image feature vector and $y=\left\{-\frac{1}{C-1}, 1\right\}^{C}$ the class vector that specifies the category membership of the image. In particular, the sign of component $y_{c}$ gives the positive/negative membership of the example to class $c(c=1,2, \ldots, C)$, such that $y_{c}$ is negative iff the observation does not belong to class $c$, positive otherwise.

In this paper, we propose to generalize the classic $k$-NN rule to the following leveraged multiclass classifier $\boldsymbol{h}^{\ell}=\left\{h_{c}^{\ell}\right\}$ :

$$
h_{c}^{\ell}\left(\boldsymbol{x}_{q}\right)=\sum_{j=1}^{T} \alpha_{j c} K\left(\boldsymbol{x}_{q}, \boldsymbol{x}_{j}\right) y_{j c},
$$

where $h_{c}^{\ell}$ is the classification score for class $c, x_{q}$ denotes the query image, $\alpha_{j c}$ the leveraging coefficients, which provide a weighted voting rule instead of uniform voting, and $K(\cdot, \cdot)$ is the $k$-NN indicator function:

$$
K\left(\boldsymbol{x}_{i}, \boldsymbol{x}_{j}\right)=\left\{\begin{array}{cc}
1, & \boldsymbol{x}_{j} \in \mathrm{NN}_{k}\left(\boldsymbol{x}_{i}\right) \\
0, & \text { otherwise }
\end{array},\right.
$$

with $\mathrm{NN}_{k}\left(\boldsymbol{x}_{i}\right)$ denoting the set of the $k$-nearest neighbors of $\boldsymbol{x}_{i}$.

Training our classifier essentially consists in selecting the most relevant subset of training data, i.e., the so-called prototypes, whose cardinality $T$ is generally much smaller than the original number $m$ of annotated instances. The prototypes are selected by first fitting the coefficients $\alpha_{j}$, and then removing the examples with the smallest $\alpha_{j}$, which are less relevant as prototypes.

In order to fit our leveraged classification rule (3) onto training set $\mathcal{S}$, we should try to directly minimize the multiclass surrogate ${ }^{1}$ (exponential) risk, which is defined as the actual misclassification rate on the training data, as follows:

$$
\varepsilon^{\exp }\left(h_{c}^{\ell}, S\right) \doteq \frac{1}{m} \sum_{i=1}^{m} \exp \left\{-\rho\left(h_{c}^{\ell}, i\right)\right\} .
$$

\footnotetext{
${ }^{1}$ We call surrogate a function that upperbounds the risk functional we should minimize, and thus can be used as a primer for its minimization.
}

where:

$$
\rho\left(h_{c}^{\ell}, i\right)=y_{i c} h_{c}^{\ell}\left(\boldsymbol{x}_{i}\right)
$$

is the multiclass edge of classifier $h_{c}^{\ell}$ on training example $\boldsymbol{x}_{i}$. This edge measures the "goodness of fit" of the classifier on example $\left(\boldsymbol{x}_{i}, \boldsymbol{y}_{i}\right)$ for class $c$, thus being positive iff the prediction agrees with the example's annotation.

In order to solve this optimization, we propose a boosting-like procedure, i.e., an iterative strategy where the classification rule is updated by adding a new prototype $\left(\boldsymbol{x}_{j}, \boldsymbol{y}_{j}\right)$ (weak classifier) at each step $t$ $(t=1,2, \ldots, T)$, thus updating the strong classifier (3) as follows:

$$
h_{c}^{(t)}\left(\boldsymbol{x}_{i}\right)=h_{c}^{(t-1)}\left(\boldsymbol{x}_{i}\right)+\boldsymbol{\delta}_{j} K\left(\boldsymbol{x}_{i}, \boldsymbol{x}_{j}\right) y_{j c} .
$$

( $j$ is the index of the prototype chosen at iteration $t$.) Using (7) into (6), and then plugging it into (5), turns the problem of minimizing (5) to that of finding $\delta_{j}$ with the following objective:

$$
\underset{\delta_{j}}{\arg \min } \sum_{i=1}^{m} w_{i} \cdot \exp \left\{-\delta_{j} \mathrm{r}_{i j}\right\} .
$$

In (8), we have defined $w_{i}$ as the weighting factor, depending on the past weak classifiers:

$$
w_{i}=\exp \left\{-y_{i c} h_{c}^{(t-1)}\left(\boldsymbol{x}_{i}\right)\right\},
$$

and $\mathrm{r}_{i j}$ as a pairwise term only depending on training data:

$$
\mathrm{r}_{i j}=K\left(\boldsymbol{x}_{i}, \boldsymbol{x}_{j}\right) y_{i c} y_{j c} .
$$

Finally, taking the derivative of (8), the global minimization of surrogate risk (5) gives the following expression of $\delta_{j}$ :

$$
\delta_{j}=\frac{1}{2} \log \frac{\gamma \cdot \sum_{i: r_{i j}^{c}>0} w_{i}}{\sum_{i: r_{i j}^{c}<0} w_{i}},
$$

where $\gamma$ is a coefficient that compensates for the class imbalance.

We provided theoretical details and properties of our boosting algorithm in (Piro et al., 2012), as well as an extension of UNN to inherent multiclass classification in (Piro et al., 2010).

We also tried a "soft" version of the UNN classification rule, called $\mathrm{UNN}_{s}$, which considers a logistic estimator for a Bernoulli prior that vanishes with the rank of the neighbors, thus decreasing the importance of farther neighbors:

$$
\hat{p}(j)=\beta_{j}=\frac{1}{1+\exp (\lambda(j-1))} .
$$

This amounts to redefining (3) as follows:

$$
h_{c}^{\ell}\left(\boldsymbol{x}_{q}\right)=\sum_{j=1}^{T} \alpha_{j c} \beta_{j} K\left(\boldsymbol{x}_{q}, \boldsymbol{x}_{j}\right) y_{j c} .
$$

(Notice that $k$-NN indexed by $j$ are supposed to be sorted from closer to farther.) 


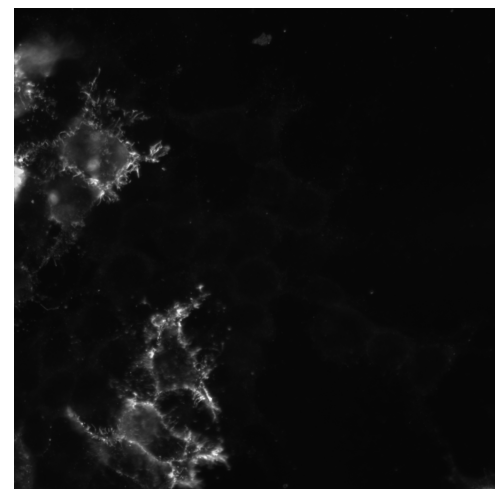

(a)

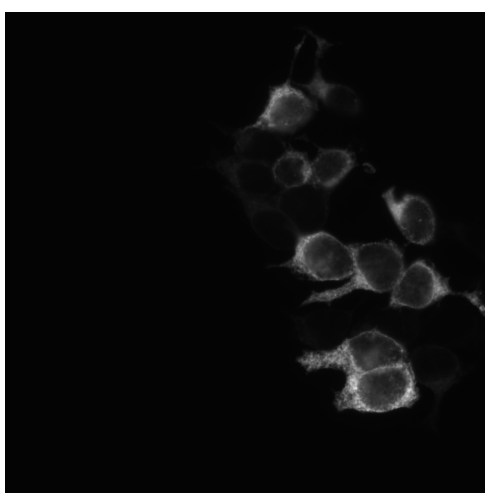

(b)

Figure 4: A sample Mb cells image (a) and ER cells image (b).

\section{EXPERIMENTS}

The images at 40-fold magnification were acquired by means of a fully fluorescence microscope (Zeiss Axio Observer Z1) coupled to a monochrome digital camera (Photometrics cascade II camera). The images have a resolution of $1024 \times 1024$ pixels,

In our biological experiments, we individually expressed different NIS proteins mutated for putative sites of phosphorylation. The effect on the protein localization of each mutation was studied after immunostaining using anti-NIS antibodies as previously described (Dayem et al., 2008). Immunocytolocalization analysis revealed three cell types with different subcellular distributions of NIS: at the plasma membrane; in intracellular compartment (mainly endoplasmic reticulum); throughout the cytoplasm (with an extensive expression).

For this purpose, we collected 489 cell images of such biological experiments and manually annotated them according to the three classes, that are denoted in the following as Mb protrusion and $M b$ (389 cells), ER (100 cells) and Round (8 cells).

Since round cells are very easy to classify (very high contrast everywhere in the cell), we focus on the two category classification: Membrane $(M b)$ and $E R$. An example of $M b$ and $E R$ cells is given in Fig. 4.

We start by extracting our features on cells images. An important parameter for our bio-inspired descriptors is the scale on which we compute the local contrast. In fact, the standard deviations of the DoG are dependant of this parameter as follows: $\sigma 1=0.5 \cdot 2^{\text {scale }-1}$ and $\sigma 2=3 \cdot \sigma 1$. We study first the more relevant scale space and the evaluations on 100 experiments are reported in the curve of the Fig. 5. Thus, according to those experiments the following evaluations are performed using the scale 5 for descriptors.

Once we get descriptors of all the cells in the

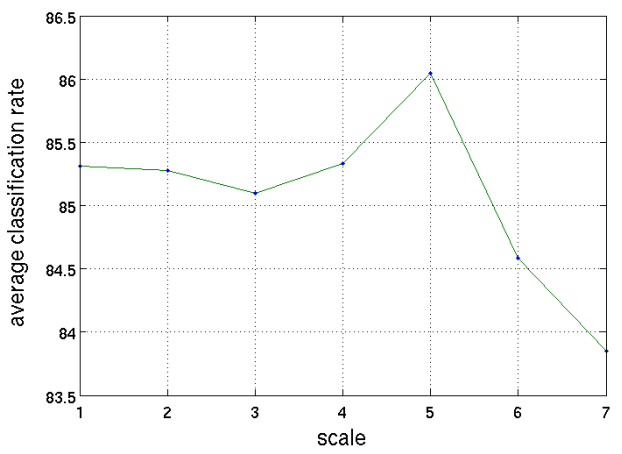

Figure 5: Average classification rate as a function of the descriptors scale using $\mathrm{UNN}_{s}$.

database, we ran our UNN algorithm by training on $50 \%$ of the images, while testing on the remaining $50 \%$. In order to get robust performance estimation, we repeated the evaluation 100 times over different random training/testing folds. Note that we used a fast and effecient tool for the $k$-NN search provided in the Yael toolbox ${ }^{2}$.

Our classification algorithm $\mathrm{UNN}_{s}$ was evaluated in a first step using a uniform regularization by the mean of the parameter $\gamma$ that compensates the class imbalance. In a second step, we focused in an adaptive regularization according to majority and minority classes and we denote this approach by $\mathrm{UNN}_{s_{-} \text {adaptive }}$. This approach allows to have automatically a balance number of trained prototypes per class (see Tab. 1) and visibly decrease misclassification.

We report the average classification results and the classification rate of each class in Tab. 2. Remark that we achieve a mean average precision (MAP) greater than $87.5 \%$ when using $\mathrm{UNN}_{\text {s_adaptive }}$, which is a very

\footnotetext{
${ }^{2}$ Source code can be downloaded in the following link: https://gforge.inria.fr/projects/yael
} 


\begin{tabular}{|l|c|c||c|c||c|c|}
\cline { 2 - 7 } \multicolumn{1}{c|}{} & \multicolumn{2}{c||}{ mAP } & \multicolumn{2}{c||}{ AP for Mb } & \multicolumn{2}{c|}{ AP for ER } \\
\cline { 2 - 7 } \multicolumn{1}{c|}{} & $\mu(m A P)$ & $\sigma(m A P)$ & $\mu(A P)$ & $\sigma(A P)$ & $\mu(A P)$ & $\sigma(A P)$ \\
\hline$k-N N$ & 84.22 & 2.56 & 94.81 & 2.02 & 73.64 & 5.63 \\
\hline $\mathrm{UNN}_{s}$ & 86.04 & 2.54 & 94.48 & 1.90 & 77.60 & 5.46 \\
\hline $\mathrm{UNN}_{\text {s_adaptive }}$ & 87.67 & 1.93 & 89.27 & 2.26 & 86.08 & 3.78 \\
\hline SVM & 76.46 & 4.55 & 95.58 & 2.38 & 57.34 & 10.67 \\
\hline
\end{tabular}

Table 2: Global average precision (MAP), average precision for $M b$ and average precision for $E R$ for different classifiers.

\begin{tabular}{|l|c|c|c|}
\cline { 2 - 4 } \multicolumn{1}{c|}{} & $N_{t}$ & $N_{M b}$ & $N_{E R}$ \\
\hline $\mathrm{UNN}_{s}$ & $69.24 \%$ & $50.20 \%$ & $19.03 \%$ \\
\hline $\mathrm{UNN}_{S_{\text {_adaptive }}}$ & $47.69 \%$ & $28.58 \%$ & $19.11 \%$ \\
\hline
\end{tabular}

Table 1: This table shows the percentage of prototypes number selected from the training set by both $\mathrm{UNN}_{s}$ and $\mathrm{UNN}_{S_{-} \text {adaptive }}$ : We report the total number $\left(N_{t}\right)$, the one in the class $M b\left(N_{M b}\right)$, and in the class $E R\left(N_{E R}\right)$. The distribution of selected prototypes on both classes is more balanced using $\mathrm{UNN}_{\text {S_adaptive }}$.

promising result for our cell descriptor and classification method. Our classification approach improves the MAP of the $k$-NN classifier of more $3 \%$ and the SVM one of more than $11 \%$. Moreover some misclassification arises on the minority class $(E R)$ using $k$-NN , thus giving an average precision (AP) of about

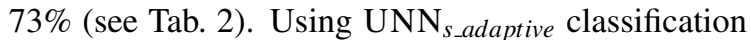
improved MAP of the minority class up to $86 \%$ thus $13 \%$ better than $k$-NNFor the SVM classification, the result in Tab. 2 shows that there is an important classification error on ER cells where the AP is about only $57 \%$.

\section{CONCLUSIONS}

In this paper, we have presented a novel algorithm for automatic segmentation and classification of cellular images based on different subcellular distributions of the NIS protein. First of all, our method relies on extracting highly discriminative descriptors based on bio-inspired histograms of Difference-of-Gaussians (DoG) coefficients on cellular regions. Then, we propose a supervised classification algorithm, called UNN, for learning the most relevant prototypical samples that are to be used for predicting the class of unlabeled cellular images according to a leveraged $k$ $\mathrm{NN}$ rule. We evaluated UNN performances on a significantly large database of cellular images that were manually annotated. Although being the very early results of our methodology for such a challenging application, performances are really satisfactory (average global precision of $87.5 \%$ and MAP of the minority class up to $86 \%$ ) and suggest our approach as a valuable decision-support tool in cellular imaging.

\section{REFERENCES}

Bel haj ali, W., Debreuve, E., Kornprobst, P., and Barlaud, M. (2011). Bio-Inspired Bags-of-Features for Image Classification. In International Conference on Knowledge Discovery and Information Retrieval (KDIR 2011).

Dayem, M., Basquin, C., Navarro, V., Carrier, P., Marsault, R., Chang, P., Huc, S., Darrouzet, E., Lindenthal, S., and Pourcher, T. (2008). Comparison of expressed human and mouse sodium/iodide symporters reveals differences in transport properties and subcellular localization. Journal of Endocrinology, 197(1):95-109.

Field, D. J. (1994). What is the goal of sensory coding? Neural Computation, 6(4):559-601.

Lowe, D. G. (2004). Distinctive image features from scaleinvariant keypoints. International Journal of Computer Vision, 60(2):91-110.

Oliva, A. and Torralba, A. (2001). Modeling the shape of the scene: A holistic representation of the spatial envelope. International Journal of Computer Vision, 42:145-175. 10.1023/A:1011139631724.

Peyrottes, I., Navarro, V., Ondo-Mendez, A., Marcellin, D., Bellanger, L., Marsault, R., Lindenthal, S., Ettore, F., Darcourt, J., and Pourcher, T. (2009). Immunoanalysis indicates that the sodium iodide symporter is not overexpressed in intracellular compartments in thyroid and breast cancers. European Journal of Endocrinology, 160(2):215-25.

Piro, P., Nock, R., Nielsen, F., and Barlaud, M. (2010). Multi-Class Leveraged $k$-NN for Image Classification. In Proceedings of the Asian Conference on Computer Vision (ACCV 2010).

Piro, P., Nock, R., Nielsen, F., and Barlaud, M. (2012). Leveraging k-nn for generic classification boosting. Neurocomputing, 80:3-9.

Schapire, R. E. and Singer, Y. (1999). Improved boosting algorithms using confidence-rated predictions. Machine Learning, 37:297-336.

Van Rullen, R. and Thorpe, S. J. (2001). Rate coding versus temporal order coding: what the retinal ganglion cells tell the visual cortex. Neural Comput, 13(6):12551283. 